\title{
ESTUDO DA RELAÇÃO ENTRE AS PROPRIEDADES DOS CARVÕES E A MICROESTRUTURA POROSA DOS COQUES
}

\author{
Anderson Azevedo Agra ${ }^{\prime}$ \\ Bruno Deves Flores ' \\ Anderson Nicolodi ' \\ Guilherme Liziero Ruggio da Silva ${ }^{2}$ \\ Antônio Cesar Faria Vilela' \\ Eduardo Osório
}

\section{Resumo}

A resistência mecânica do coque metalúrgico está intimamente relacionada com sua microestrutura porosa, de forma que o conhecimento quantitativo dos parâmetros microestruturais formada pelos diferentes tipos de carvões pode ser de grande utilidade. Nesse trabalho foram produzidos coques laboratoriais a partir de carvões com diferentes propriedades. A caracterização microestrutural foi realizada a partir de uma metodologia proposta de microscopia ótica associada a análise de imagem. O estudo indica que o teor matéria volátil, fluidez e a composição maceral dos carvões são preponderantes na formação da microestrutura porosa dos coques. O carvão de alta matéria volátil e alta fluidez estudado deu origem a um coque com elevada porosidade e com poros grandes. $\mathrm{O}$ carvão ensaiado com elevada quantidade de macerais do grupo da inertinita gerou coque com microestrutura de baixa porosidade, no entanto com grande concentração de poros de baixa circularidade. O carvão e a mistura com conteúdo de matéria volátil e fluidez intermediários deram origem a coques com microestruturas porosas compostas por poros de pequenas dimensões e com formas mais adequadas.

Palavras-chave: Coque metalúrgico; Microestrutura; Porosidade; Carvão.

\section{STUDY OF THE RELATIONSHIP BETWEEN COAL PROPERTIES AND COKE MICROSTRUCTURE}

\begin{abstract}
The mechanical strength of metallurgical coke is closely related to its porous microstructure, so that the quantitative knowledge of the microstructural parameters formed by the different types of coals can be very useful. In this work laboratory cokes were produced from coals with different properties. The microstructural characterization was performed from a proposed methodology of optical microscopy associated with image analysis. The study indicates that the volatile matter content, fluidity and the maceral composition of the coals are preponderant in the formation of the porous microstructure of the cokes. Coals of high volatile matter and high fluidity studied formed cokes with high porosity and large pores. The coal with high content of the inertinite maceral group gave rise to a coke with microstructure of low porosity, although with great concentration of pores of low circularity. The coal and blend with volatile matter content and fluidity intermediate formed cokes with microstructure composed by pores of small sizes and more suitable forms.
\end{abstract}

Keywords: Coke; Microstructure; Porosity; Coal.

\section{INTRODUÇÃO}

O coque metalúrgico exerce diversas funções dentro do alto-forno, dentre elas, o papel estrutural de sustentação de carga tem especial importância, visto que é a única matéria-prima empregada para tal objetivo. A resistência mecânica do coque está intimamente vinculada com a microestrutura porosa desse material, que pode ser definida como a distribuição espacial da matriz carbonosa do coque e a sua porosidade. As relações entre parâmetros microestruturais do coque e sua resistência mecânica foi demostrada em diversos trabalhos [1-6]. A microestrutura porosa é gerada no intervalo plástico dos carvões no processo de coqueificação. Por essa razão as propriedades

'Laboratório de Siderurgia - LaSid, Universidade Federal do Rio Grande do Sul - UFRGS, Porto Alegre, RS, Brasil. E-mail: anderson.agra@ufrgs.br ${ }^{2}$ Gerdau Ouro Branco, Ouro Branco, MG, Brasil. 
dos carvões têm grande influência na microestrutura resultante do coque $[2,6]$. O presente trabalho tem como objetivo verificar o efeito das propriedades de carvões nos parâmetros microestruturais de coques laboratoriais obtidos a partir de uma metodologia de microscopia ótica associada a análise de imagem.

\section{MATERIAIS E MÉTODOS}

\section{I Materiais}

Para esse estudo foram produzidos coques laboratoriais a partir de três amostras comerciais de carvões individuais (A, B e C) e uma amostra de mistura de carvões (D) tipicamente comercializada para produção de coque industrial. Na escolha das matérias-primas buscou-se uma variedade de propriedades, visando gerar coques com diferentes níveis de porosidade e outros parâmetros microestruturais. A Tabela I apresenta alguns dados de interesse da caracterização dos materiais.

\subsection{Preparação de Amostras para Microscopia}

As análises microestruturais foram realizadas a partir de coques produzidos com os carvões/mistura da Tabela I em escala laboratorial, conforme metodologia descrita na referência Agra et al. [7]. A partir de cada amostra foram selecionadas cinco partículas com granulometria entre 19 e $22,4 \mathrm{~mm}$ (granulometria tipicamente utilizada no ensaio de CRI/CSR). Essas partículas foram embutidas em resina epóxi e a obtenção e a preparação da superfície para microscopia foi realizada a partir de lixamento (lixas de granulometria $80,180,400,600$ e I200) e posterior polimento (pano de veludo com suspensão de alumina 0,5 e 0,03 $\mu \mathrm{m}$ ).

\subsection{Obtenção e Composição das Imagens}

A captura das imagens foi realizada em microscópio ótico Leica DM6000 M utilizando aumento de 50x. As imagens obtidas têm dimensões de 2500 x $1800 \mu \mathrm{m}$ com resolução de 0,544 pixels $/ \mu \mathrm{m}$. Para cada plug foram capturadas 20 imagens em sequência no plano $X-Y$, utilizando uma mesa motorizada de forma a possibilitar a composição de uma única imagem com uma grande área do coque, porém com alta resolução. A composição das imagens foi realizada utilizando o software
Adobe Photoshop, conservando a resolução das imagens individuais. Para a análise das imagens foi utilizado $56,7 \mathrm{~mm}^{2}$ (4100 pixels $^{2}$ ) de área proveniente de cada plug.

\subsection{Algoritmo de Análise das Imagens em ImageJ}

Para análise das imagens dos coques foi utilizado o software de código aberto Imagej. Uma sequência de comandos foi desenvolvida e gravado em uma macro, facilitando o acesso dos comandos e automatizando o processo. Na Figura I é apresentado esquematicamente o algoritmo para a análise das imagens.

$\mathrm{Na}$ imagem de entrada (Figura IA) é possível visualizar uma região de coloração mais clara, correspondente ao material carbonoso (parede do coque) e regiões em cinza e preto que representam os poros, com e sem preenchimento de resina, respectivamente. A segmentação Threshold é utilizada para separação do primeiro plano e plano de fundo, produzindo uma imagem binária (Figura IB). A segmentação por Watershed, para o contexto desse trabalho, foi aplicada para definir os contornos dos poros a serem medidos (linhas amarelas na Figura IC).

O software fornece dados da amostra global (Figura ID) e também individual para a lista dos poros avaliados. Nessa metodologia foi escolhido não realizar a análise de poros com área inferior a $8000 \mu \mathrm{m}^{2}$ (área equivalente a um círculo de aproximadamente $100 \mu \mathrm{m}$ de diâmetro), uma vez que são justamente os poros com área superior a este valor os mais influentes na resistência mecânica do coque [5]. O volume dos poros com tamanho inferior a $100 \mu \mathrm{m}$ somam cerca de $5 \%$ do volume total dos poros do coque, sendo função do tipo de carvão que lhe deu origem.

A partir dos parâmetros obtidos pode-se realizar uma classificação dos poros em termos de qualidade (Figura IE). Essa separação foi realizada com base em estudos que apontam que poros com diâmetro equivalente $\left(D_{\text {eq }}\right)$ superior a $300 \mu \mathrm{m}$ e circularidade $(C)$ inferior a 0,2 são os mais críticos para a resistência mecânica $[3,4,6]$. Por último pode-se realizar um mapeamento desses poros críticos e gerar uma imagem como a Figura IF, onde as regiões em branco representam as paredes do coque, azul poros de tamanho e forma regulares e vermelho poros críticos pelos critérios citados. As regiões em preto são áreas não analisadas.

Tabela I. Caracterização dos carvões e misturas utilizados para produção dos coques laboratoriais

\begin{tabular}{|c|c|c|c|c|c|c|c|c|c|}
\hline Coques & Carvões & $M^{\text {(bs) }}$ & $\mathrm{Cz}^{\text {(bs) }}$ & $\mathbf{R m}$ & $\mathbf{V}^{(\% \mathrm{vol})}$ & $\mathbf{L}^{\text {(\%vol) }}$ & I\%vol) & $I P^{(0 C)}$ & $\log M F^{(d d p m)}$ \\
\hline$C_{A}$ & A & 34,3 & 6,8 & 0,84 & 74,6 & 9,6 & $\mathrm{II}, 4$ & 84 & 4,1 \\
\hline $\mathrm{C}_{\mathrm{B}}$ & B & 20,5 & 9,6 & 1,16 & 58,0 & 0,0 & 32,0 & 44 & 0,5 \\
\hline Cc & C & 20,6 & 9,5 & $|, 2|$ & 75,4 & 0,4 & 17,0 & 77 & 2,5 \\
\hline$C_{D}$ & D & 25,2 & 8,8 & 1,02 & 72,8 & 0,8 & 18,8 & 78 & 2,4 \\
\hline
\end{tabular}

MV = Matérial volátil; $\mathrm{Cz}=$ Cinzas; bs = base seca; \%vol = percentual volumétrico; $\mathrm{Rm}=$ refletância média da vitrinita; $\mathrm{V}=$ vitrinita; $\mathrm{L}=$ liptinita; $\mathrm{I}=$ inertinita; IP = intervalo plástico; MF = Máxima Fluidez; ddpm = divisão de dial por minuto. 


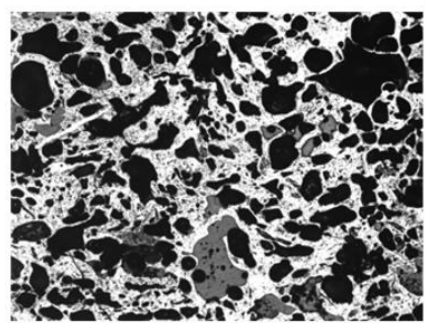

(A) IMAGEM DE ENTRADA

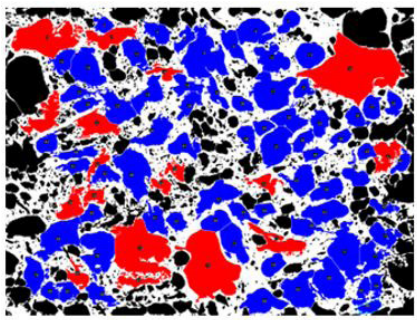

(F) MAPEAMENTO DOS POROS CRÍTICOS A RESISTÊNCIA MECÂNICA

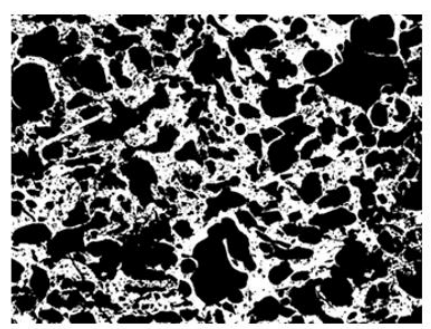

(B) SEGMENTAÇÃO THRESHOLD

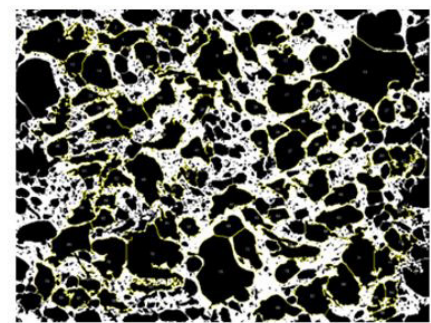

(C) SEGMENTAÇÃO WATERSHED

- Diâmetro equivalente $>300 \mu \mathrm{m}$

$$
\mathrm{D}_{\mathrm{eq}}=\sqrt{\frac{4 . A}{\pi}}
$$

- Circularidade $<0,2$

$$
\mathrm{C}=\frac{4 \pi x A}{P^{2}}
$$

\begin{tabular}{|c|c|}
\hline Nome & Coque \\
\hline Número de poros $\left(\mathrm{mm}^{2}\right)$ & 15 \\
\hline Tamanho médio $\left(\mu \mathrm{m}^{2}\right)$ & $2.64 \mathrm{E}+04$ \\
\hline Porosidade (\%) & 40.5 \\
\hline Perímetro médio $(\mu \mathrm{m})$ & $1.50 \mathrm{E}+03$ \\
\hline
\end{tabular}

(E) ANÁLISE DO TAMANHO DOS POROS E CIRCULARIDADE
(D) PARÂMETROS MICROESTRUTURAIS

Figura I. Representação dos passos da metodologia de caracterização da microestrutura porosa de coque por análise de imagem dos coques.

\section{RESULTADOS}

Os coques laboratoriais produzidos a partir dos carvões apresentados na Tabela I tiveram suas microestruturas caracterizadas através da metodologia apresentada nos tópicos anteriores. O resultado dessas caracterizações é apresentado na Tabela 2 e o mapeamento dos poros críticos a resistência mecânica é mostrado na Figura 2.

O coque do carvão de alta matéria volátil $(A)$ apresentou a microestrutura com menor número de poros por área, resultado do maior tamanho dos seus poros (maior diâmetro equivalente). $O$ coque $C_{B}$, por sua vez, apresentou a menor porosidade, maior número de poros por área e consequentemente o menor tamanho de poros entre os coques individuais analisados. $\mathrm{O}$ coque $\mathrm{C}_{\mathrm{c}}$ apresentou porosidade e tamanho médio de poros intermediário entre os coques dos $C_{A}$ e $\circ C_{B}$. $O$ coque produzido a partir da mistura de carvões $D$ apresentou porosidade maior que os coques dos carvões de média material volátil, porém inferior à do carvão de alto volátil. $O$ tamanho médio dos poros, no entanto, apresentou-se intermediário entre os carvões de médio volátil analisado, com diâmetro equivalente de $2 / 2,5 \mu \mathrm{m}$.

Na Tabela 2 é apresentado também a caracterização dos poros críticos para a resistência mecânica, determinado pelos critérios citados anteriormente. O coque com maior percentual volumétrico de poros críticos é o coque $C_{A}$,
Tabela 2. Caracterização microestrutural dos coques laboratoriais

\begin{tabular}{ccccccc}
\hline \multirow{2}{*}{ Coques } & \multirow{2}{*}{$\mathbf{N}^{(\text {poros } / \mathrm{mm} 2)}$} & \multirow{2}{*}{$\mathbf{P}^{(\%)}$} & \multirow{2}{*}{$\mathbf{D}_{\text {eq }}{ }^{\mu \mu \mathrm{m})}$} & \multicolumn{3}{c}{ Área } \\
\cline { 5 - 7 } & & & $\mathbf{D}_{\text {eq }}>300 \mu \mathrm{m}$ & $\mathbf{C}^{<0,2}$ & $\mathbf{T C}$ \\
\hline $\mathbf{C}_{\mathbf{A}}$ & 12,0 & 68,7 & 254,5 & 61,7 & 3,9 & 63,0 \\
$\mathbf{C}_{\mathbf{B}}$ & 15,9 & 55,4 & 204,9 & 33,0 & 27,4 & 47,8 \\
$\mathbf{C}_{\mathrm{C}}$ & 15,5 & 61,0 & 220,7 & 43,7 & 4,9 & 45,6 \\
$\mathbf{C}_{\mathrm{D}}$ & 17,2 & 65,0 & 212,5 & 40,8 & 16,0 & 48,1 \\
\hline
\end{tabular}

$\mathrm{N}=$ Número de poros por área; $\mathrm{P}=$ Porosidade; $\mathrm{Deq}=$ diâmetro equivalente; $\mathrm{C}=$ circularidade; $\mathrm{TC}=$ total de poros críticos.

onde $63,0 \%$ da área ocupada por poros correspondem a poros críticos. Nota-se que a maior contribuição para esses poros críticos ocorre por poros de área excessiva. Os coques de média matéria volátil, $\mathrm{C}_{\mathrm{B}}$ e $\mathrm{C}_{\mathrm{C}}$, apresentaram os menores percentuais volumétricos ocupado por poros críticos sendo eles 47,8 e $45,6 \%$, respectivamente. Observa-se que a maior quantidade de poros críticos por baixa circularidade foi observada para o coque $C_{B}$. A análise do coque de mistura $C_{D}$ mostrou que sua microestrutura apresenta porcentual volumétrico ocupado por poros críticos intermediário entre os coques individuais de média e alta matéria volátil, sendo majoritariamente compostos por poros de área excessiva. 


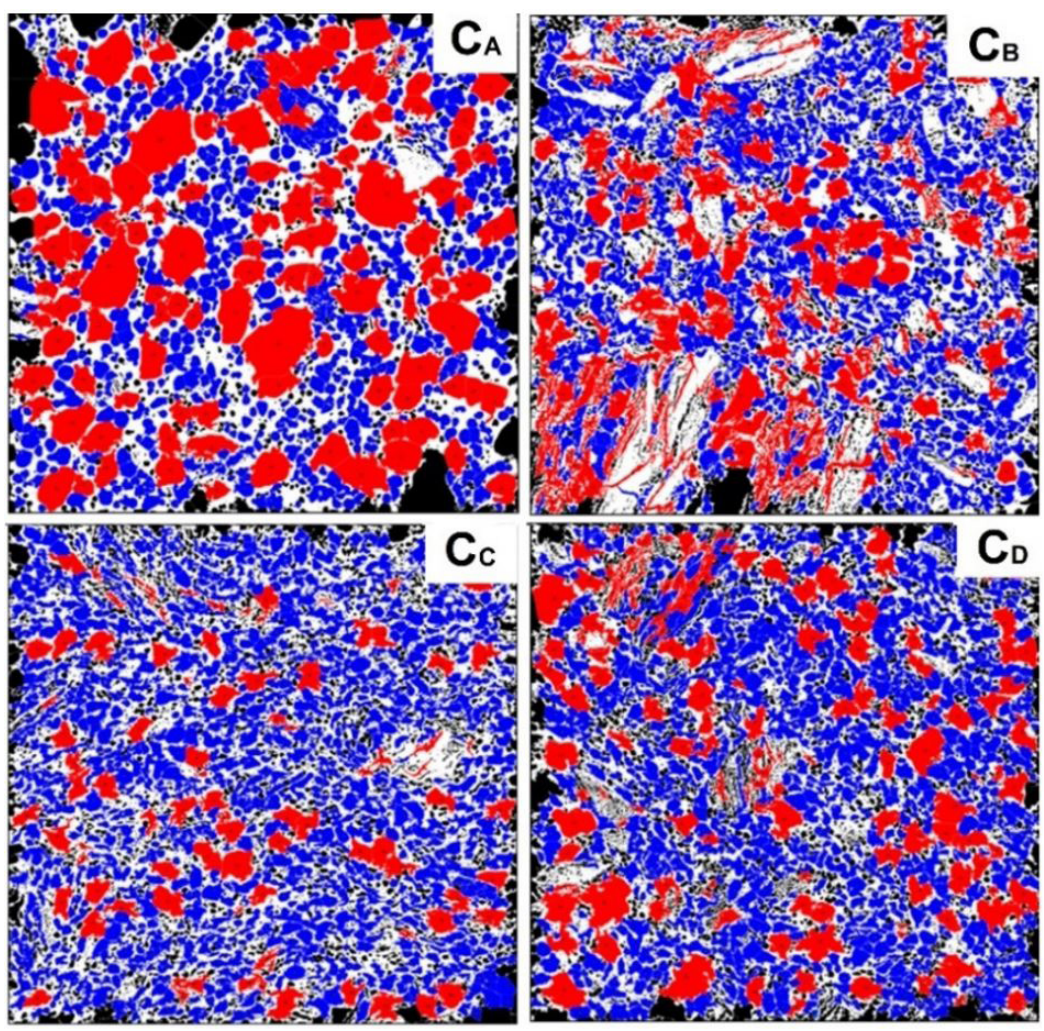

Figura 2. Mapeamento dos poros críticos para a resistência mecânica dos coques laboratoriais.

\section{DISCUSSÃO}

Conforme apresentado na Tabela I o carvão de alto volátil A possui propriedades termoplásticas extremamente desenvolvidas. Estudos que buscaram relacionar termoplasticidade de carvões individuais e qualidade de coque mostram que os carvões com fluidez superior a $1000 \mathrm{ddpm}$ não produzem coques com resistência mecânica adequada, devido a excesso de plasticidade estar associado a formação de microestrutura demasiadamente porosa e com poros grandes [8]. Isso pode ser quantificado na Tabela 2, e pode ser observado na Figura 2 nas regiões em vermelho. $O$ coque $C_{B}$, produzido a partir de um carvão de médio volátil, e que possui poucas propriedades termoplásticas (Tabela I), apresenta a mais baixa porosidade entre os coques de carvões individuais do estudo. Isso se deve ao elevado conteúdo do grupo maceral da inertinita presente na composição maceral desse carvão. Os macerais desse grupo não colaboram para a produção de metaplasto no processo de coqueificação e ainda atuam adsorvendo o mesmo que está presente no sistema. Como consequência ocorre redução significativa da plasticidade [9-10]. Os componentes inertes desses coques podem ser distinguidos na Figura 2 pelo seu relevo e bordas com ângulos retos, típico de materiais não fundidos. Carvões com elevado conteúdo de macerais inertes produzem coques com alta densidade de interfaces matriz/inerte, reduzindo significantemente a resistência mecânica [3,4]. A caracterização da microestrutura do coque produzido a partir do carvão $\mathrm{Ce}$ da mistura de carvões $D$ apresentaram os melhores valores dos parâmetros analisados. Isso pode ser observado pelo predomínio da região em azul na Figura 2 para esses coques. Essa boa qualidade microestrutural pode ser relacionada com as propriedades dos carvões de origem, conforme já foi avaliado em trabalhos anteriores, carvões com valor de fluidez entre 200 e $1000 \mathrm{ddpm}$ produzem coques de boa qualidade [8].

\section{CONCLUSÕES}

O presente estudo mostrou que a metodologia proposta de microscopia ótica associado a análise de imagens é capaz de caracterizar a microestrutura porosa de coques metalúrgicos. Foi demonstrado que as propriedades dos carvões, como matéria volátil, fluidez e composição maceral, governam a microestrutura porosa dos coques formados. O carvão com alta matéria volátil e alta fluidez estudado deu origem a um coque de elevada porosidade e com poros de grandes dimensões. O carvão com alto conteúdo de macerais do grupo da inertinita gerou um coque com microestrutura de baixa porosidade, no entanto com grande concentração de poros de baixa circularidade. O carvão e a mistura ensaiadas de matéria volátil e fluidez intermediária produziram microestruturas porosas mais adequadas, com poros de pequenas dimensões e formas mais regulares.

\section{Agradecimentos}

Os autores desse trabalho agradecem a Gerdau Usina Ouro Branco pelo fornecimento dos carvões importados e o Conselho Nacional de Desenvolvimento Científico e Tecnológico (CNPq) pelo incentivo financeiro. 


\section{REFERÊNCIAS}

I. Patrick JW, Walker A. Macroporosity in cokes: its significance, measurement, and control. Carbon. 1989;27(I): I I7123.

2. Sato $\mathrm{H}$, Patrick JW, Walker A. Effect of coal properties and porous structure on tensile strength of metallurgical coke. Fuel. 1998;77(II):I203-I 208.

3. Andriopoulos N, Loo CE, Dukino R, McGuire S. Micro-properties of australian coking coals. ISIJ International. 2003;43(10): I528-1537.

4. Kubota Y, Nomura S, Arima T, Kato K. Quantitative evaluation of relationship between coke strength and pore structure. Tetsu-To-Hagane. 2010;96(5):328-336.

5. Donskoi E, Poliakov A, Mahoney MR, Scholes O. Novel optical image analysis coke characterisation and its application to study of the relationships between coke structure, coke strength and parent coal composition. Fuel. 2017;208:28I-295.

6. Meng, Fanyu Et Al. Characterization of microstructure and strength of coke particles and their dependence on coal properties. Powder Technology. 2017;320:249-256.

7. Agra AA, Flores BD, Nicolodi A, Silva GLR, Vilela AC, Osório E. Microestrutura do coque: desenvolvimento de análise quantitativa via microscopia ótica associada a análise de imagem. In: Associação Brasileira de Metalurgia, Materiais e Mineração. Anais do $48^{\circ}$ Seminário de Redução de Minérios e Matérias-primas; 2018; São Paulo, Brazil. São Paulo: ABM Week; 2018.

8. Díez MA, Alvarez R, Barriocanal C. Coal for metallurgical coke production: predictions of coke quality and future requirements for cokemaking. International Journal of Coal Geology, 2002;50(I-4):389-4I 2.

9. Fernández AM, Barriocanal C, Díez MA, Alvarez R. Importance of the textural characteristics of inert additives in the reduction of coal thermoplastic properties. Fuel. 2010;89(I I):3388-3392.

I0. Loison R, Foch P, Boyer A. Coke: quality and production. 2nd ed. Paris: Ed. Butterworths; 1989.

Recebido em: 30 Nov. 2018

Aceito em: 23 Ago. 2019 